\title{
Transformaciones en las subjetividades de los trabajadores: casos de empresas colombianas recuperadas"
}

\author{
Transformations in Workers' Subjectivities: Cases from \\ Recovered Colombian Companies
}

Recibido: agosto 6 de 2009 | Revisado: enero 27 de 2010 | Aceptado: junio 17 de 2010

\author{
Olga LuCÍA HuERTAS ** \\ RicARDo DÁvila LADRÓN DE GUEVARA **: \\ DARÍO CASTILLO ***** \\ Pontificia Universidad Javeriana, Bogotá, Colombia
}

\section{RESUMEN}

Este artículo presenta los resultados de un proyecto de investigación interdisciplinar que exploró las trasformaciones en la subjetividad en cuatro empresas colombianas que, luego de haberse enfrentado a una crisis económica o a la quiebra, fueron entregadas a sus trabajadores para ser recuperadas. La metodología utilizada fue el de estudio de caso; se revisaron documentos de fuentes primarias y secundarias, se realizaron entrevistas a semiestructuradas y grupos focales. Los resultados permitieron evidenciar que frente a esta experiencia, en todos los casos, los trabajadores transformaron las comprensiones que tenían sobre ellos mismos y sus relaciones sociales, pero solo en uno de ellos se pudo observar una revalorización de su condición de trabajador.

Palabras clave autores

Empresas recuperadas, Psicología del trabajo, subjetividad, trabajadores,

investigación cualitativa.

Palabras clave descriptores

Psicología ocupacional, relaciones laborales, actitud laboral, calidad de la vida

laboral, delegación de autoridad, liderazgo, estatus profesional.

\section{A B S T R A C T}

This paper presents the results of an interdisciplinary research project that explored the subjectivity's changes of workers, in the four Colombian companies that after has an economical crisis or insolvency, was delivered to their workers to be recovered. The methodology used was the case study, reviewed documents of primary and secondary sources, in-depth interviews and focus groups. The results show that compared to this experience in all cases, workers had transformed understandings about themselves and their social relations, but only one of them saw a revaluation of its status as a worker. Key words authors

Recovered Companies, Work Psychology, Subjectivity, Workers, Qualitative Research.

Key words plus

Occupational Psychology, Labour Relations, Work Attitudes, Quality of Working Life, Delegation of Authority, Leadership, Occupational Status. 


\section{Introducción}

La investigación realizada buscó revisar, de manera interdisciplinaria y con una orientación cualitativa, algunos casos colombianos que se pueden entender dentro de la experiencia de las empresas recuperadas en América Latina. El presente artículo pretende mostrar privilegiadamente los resultados frente a la categoría de subjetividad, especialmente, al objetivo de reconocer las transformaciones en las subjetividades de los trabajadores (en su mayoría obreros de la producción) que han atravesado por la experiencia de recuperar una empresa, en cuatro organizaciones colombianas vinculadas a la investigación.

En la investigación se exploró la categoría subjetividad, entendida desde las aproximaciones de las psicologías construccionistas y críticas; en particular, se retomó el trabajo de Cabruja y Garay (2005). Desde estas perspectivas, la comprensión de la subjetividad de los trabajadores dio cuenta, especialmente, de los sentidos construidos sobre la experiencia de quiebra y la creación de una nueva empresa, de las transformaciones que han experimentado como trabajadores y como socios, de la comprensión sobre cómo han sido ellos entendidos en las relaciones generadas por la liquidación de la Compañía, en su condición histórica de trabajadores y de la emergencia de su nueva condición de empresarios.

La segunda categoría explorada fue Estrategias de Gestión, la cual fue entendida como el conjunto de medidas tomadas por una persona u organización, a partir de una situación inicial y una información disponible para el logro de una meta pretendida, que con frecuencia son una respuesta emergente a circunstancias no previstas o planeadas en un momento determinado. Los resultados referentes a la categoría Estrategias de Gestión pueden consultarse en Dávila, Huertas y Castillo (2010).

\section{Elementos para comprender las posibilidades de transformación de las subjetividades}

A continuación, se realizará una breve revisión de cuatro elementos que servirán para comprender las posibilidades de transformación de las subjetividades de los involucrados en la experiencia de recuperar una empresa. Los elementos propuestos son: la comprensión de las crisis y quiebras como oportunidades, la comprensión de la subjetividad, las implicaciones de ser obrero en Colombia y la experiencia de recuperar empresas en otros países.

\section{Las crisis y quiebras empresariales ¿son oportunidades?}

Las organizaciones de trabajo contemporáneas experimentan actualmente, más que en otras épocas, la posibilidad de enfrentarse a la crisis (Ferrari, 2008); esto representa una situación que pone en evidencia las capacidades que pueden tener y desarrollar las empresas, los empresarios y sus trabajadores para afrontar eventos inesperados y críticos. Para los trabajadores poco calificados o los pequeños empresarios, mantener el control de todas las variables implicadas en la situación de crisis y asumir la incertidumbre no siempre resulta ser posible, por lo que algunos terminan experimentando sentimientos paradójicos y de confusión (Huertas, 2007; Serrano, Moreno \& Crespo, 2001). Es importante rescatar que el afrontamiento de dichas situaciones no depende solamente de la voluntad de los involucrados, sino también de los recursos disponibles tanto materiales (capacidad de producción, maquinaria o bienes disponibles, recursos económicos - incluidos créditos-) como de los recursos intangibles de la empresa (capital social, conocimiento) y de las oportunidades en el mercado.

También es importante señalar que cuando una empresa entra en crisis, la confianza de los socios, de los trabajadores y de los actores externos a la empresa, se debilita; estos pueden llegar a considerar que la condición de incertidumbre que se afronta no provee la suficiente seguridad para 
realizar inversión alguna en ella, ya sea de carácter económico o más agudamente la inversión de un proyecto vital personal (Sennet, 2006). Como consecuencia de estas circunstancias, las empresas en crisis económica o en quiebra comienzan a tener dificultades para obtener créditos que les ayuden a mejorar o generar alguna inversión que les permita salir de su situación; mientras, paralelamente, algunos trabajadores y socios pueden considerar la idea de retirarse para buscar horizontes que les permitan mayor bienestar o perspectiva a sus proyectos vitales.

No obstante, existen experiencias en las cuales los trabajadores terminan haciéndose cargo de la empresa y convirtiéndose en sus dueños, lo que les puede otorgar la posibilidad de generar condiciones de movilidad social y nuevas oportunidades (Bialakowsky, Grima, Costa \& López, 2005). Oportunidades que pueden evidenciarse tanto en elementos de carácter objetivo, tangibles y cuantificables (mejores ingresos, nuevos accesos, mayor consumo) como en dimensiones subjetivas, las cuales tienen que ver con la forma en que los trabajadores perciben estos cambios en la búsqueda de su bienestar (Graham \& Pettinato, 2004).

\section{Aproximaciones a la subjetividad}

Al trabajo se le ha reconocido un papel importante en la vida social y en la conformación de las personas como tales. Aunque ahora con menos frecuencia, pero aún de manera hegemónica, nos seguimos definiendo desde la labor que desempeñamos: soy arquitecto, soy profesor, soy desempleado. Esta utilización en el mundo de las relaciones del "soy", más que del "estoy" o "estoy siendo" como posibilidades transitorias para pensar la vida, se convierte en un determinante social del quienes somos. La categoría subjetividad se enmarca dentro de las aproximaciones teóricas que comprenden al sujeto como una construcción social, es decir un sujeto historizado producto de las relaciones sociales, políticas y económicas en las cuales se encuentra inmerso (Fernández, 2005). La categoría puede ser comprendida solo cuando se traspasan las fronteras de las mismas disciplinas; en este sentido, no es posible comprender que quienes somos sea un producto del resultando de una elección dicotómica entre el desarrollo de la psique en su tránsito por los procesos evolutivos o el determinismo cultural de los grupos a los cuales pertenecemos, sino que más bien se trata de una condición de interacción en permanente tensión.

González (2002) y Fernández (2005) esbozan algunos aspectos del término subjetividad. En este recorrido, señalan la necesidad de desplazar la comprensión del sujeto de las ciencias modernas hacia una perspectiva más histórico-crítica.

En este sentido, para González (2002) la subjetividad es un "(...) sistema complejo de significaciones y sentidos subjetivos producidos en la vida cultural humana (...) [es un] sistema procesual, plurideterminado, contradictorio, en constante desarrollo y sensible a la cualidad de sus momentos actuales, el cual tiene un papel esencial en las diferentes opciones del sujeto" (p. 24), por lo que la experiencia y el sentido construido a partir de ella, juegan un papel organizador en la subjetividad.

Este carácter novedoso de la noción de subjetividad se muestra en las rupturas que pretende desarrollar, frente a la tradición de la Psicología científica, en su búsqueda por encontrar la esencia del sujeto. Una profunda dimensión histórica de la subjetividad implicará reconocer en acuerdo con Cabruja y Garay (2005) que "el conocimiento está en los patrones de la relación social, en los espacios intersubjetivos, y que llevar a cabo una reflexión y deconstrucción continúa, busca circunscribir el conocimiento a unas condiciones socio-históricas de producción determinadas" (p. 44). Describir la diversa y compleja naturaleza cualitativa de la subjetividad, resulta siendo limitada cuando se utilizan instrumentos estandarizados o de respuestas simples o cerradas, por lo que nos vemos en la obligación de dar cuenta de las relaciones que se generan cuando el sujeto se enfrenta a determinados hechos.

En el marco del presente trabajo, la subjetividad se entenderá como "una constitución socio-histórica de quién somos, la cual da cuenta de la manera como se experimenta nuestro yo (...) retomando a Foucault, se comprenderá como la manera en que el sujeto lleva a cabo la experiencia de sí, en 
un juego de verdad en el que tiene relación con él mismo" (Cabruja \& Garay, 2005, p. 48). En este mismo sentido, Halford y Leonard (2006) rescatan el papel que juegan las nociones de lugar, espacio y tiempo a la hora de contextualizar las subjetividades que se construyen en el espacio de trabajo.

\section{Ser obrero en Colombia}

En Latinoamérica, existen diferentes estudios que hablan sobre la clase obrera. Resulta interesante destacar que dichos estudios han sido realizados sobre todo por historiadores, sociólogos del trabajo y antropólogos. En Colombia, son reconocidos los trabajos de Archila (1991), Weiss (1997) y Arango (1992). El término obrero comenzó a usarse a finales del siglo XIX; para esta época, Archila lo describe como "el trabajador manual de los talleres artesanales, que no necesariamente cuenta con una relación salarial" (p. 385). Las identidades obreras emergentes fueron una mezcla de las relaciones entre los campesinos y sus familias de origen, los artesanos de las incipientes ciudades de finales del siglo XIX y la región donde desempeñaban la actividad productiva (Archila, 1991; Castañeda, 1997). La mirada por parte de las elites o clases dominantes y del resto de la sociedad sobre el obrero, lo identificaron con el estrato bajo del mundo artesanal, llegando incluso a identificar al obrero con el "pobre".

Para los años 40, la población económicamente activa estaba constituida, mayoritariamente, por hombres jóvenes, a excepción de la rama manufacturera donde era posible encontrar un gran número de mujeres que se habían incorporado, como manera de apoyar la economía familiar. Arango (1992) también profundiza en la comprensión de la vinculación de mujeres a la industria nacional y, a partir de la historia de Fabricato, describe las políticas de gobierno sobre las obreras. Logra caracterizar diferentes prácticas como el "paternalismo cristiano", que buscaban generar mayor control sobre el obrero en los mundos públicos y privados; estas prácticas terminaron por generar obreros sumisos y pasivos que se adhirieron más fácilmente al control para la producción.
Otra característica de estas primeras generaciones obreras era el bajo nivel de educación formal, lo que se conoce como mano de obra poco calificada. No obstante, por el mismo desarrollo de las industrias y la segmentación de los mercados de trabajo, la calificación fue más bien empírica en el puesto de trabajo y en los procesos de socialización (Castañeda, 1997). Durante los años de 1950 a 1960, la entrada del SENA (Servicio Nacional de Aprendizaje) y de nuevas formas de gestión de las empresas, transforman en algunos casos la relación paternalista que se había establecido (Dávila, 2001), cambiando las políticas de manejo con los trabajadores. Se establecen mecanismos de subordinación y control basados en la protección y formación de los trabajadores, de tal manera que este se ajuste a los rápidos procesos de modernización (Pulido, 2007).

En un estudio más reciente, Castañeda (1997) realizó una caracterización del obrero en la industria nacional, en la cual señala la diversidad de ser obrero, donde es posible reconocer un proceso de diferenciación social entre los mismos trabajadores. Atributos como "la edad, los ciclos de vida, el sexo de los trabajadores influyen de manera decisiva para generar diferencias entre ellos, ya no sólo en sus condiciones objetivas de mercado, sino en sus actitudes y sus estrategias laborales" (p. 385). Pero ¿Qué sucede con el trabajador obrero cuando ahora accede a la propiedad de la empresa?, ¿Cómo al cambiar la regulación, las relaciones de poder y la empresa misma, se transforma el trabajador?

\section{Experiencias de empresas recuperadas por los trabajadores en Latinoamérica}

Diversas causas entre las cuales se encuentra la crisis económica y la mala fe de algunos de dueños, han llevado a un importante número de empresas a la quiebra en Argentina y Uruguay. Frente a esta situación, sus propios trabajadores se resistieron al cierre de estas a partir de la ocupación de las instalaciones (toma) o el reclamo de la propiedad para sacarlas de la quiebra, naciendo así lo que se conocen como empresas recuperadas. Luego desde el movimiento social generado a partir de 
cooperativas, se logró el acceso a la propiedad de estas, para continuar con su funcionamiento, garantizando de esta manera conservar la fuente de empleo y las vinculaciones sociales que provee el trabajo (Guerra, Martí \& Amorín, 2004; Martí, 2007). En razón a la mayor formación política de los trabajadores en estos países y a su actividad sindical, la experiencia de las empresas recuperadas se expresa, según Fontela (2007), en tres situaciones que conviene resaltar: " a) la concreción de una propuesta de organización de la experiencia social y política; b) la definición de ejes conceptuales para implementar un debate de mayor profundidad sobre la experiencia vivida y c) la determinación de situaciones a tener en cuenta para una definición amplia de empresa recuperada" (p. 87).

La manera como los trabajadores se hicieron cargo de la empresa, involucró iniciativas colectivas de los sindicatos y grupos de trabajadores, para evitar el cierre de las fábricas que se declaraban en quiebra. A través de la ocupación o toma de la empresa y de la presión para que el Estado expropiara a los empresarios de aquellas empresas que estaban en quiebra o que amenazaban, por razones económicas, cesar su actividad, esta experiencia construyó un fenómeno político conocido como el Movimiento Nacional de Fábricas Recuperadas (Fontela, 2007). Varias investigaciones han documentado estas experiencias. Los trabajos de Deledicque, Féliz y Moser (2005), Bialakowsky, Grima, Costa y López (2005) y Bialakowsky, Robledo, Rosendo y Costa (2004) muestran, a través de estudios de caso, los modos de autogestión obrera que se implementaron para sacar la empresa a flote (emergieron aspectos como la eliminación de jefaturas, la participación en la toma de decisiones, el cambio en las remuneraciones y la rotación de tareas, el manejo de la cooperación y el conflicto), los cuales no sólo lograron modificar algunos aspectos de la cultura de la organización, sino que permitieron la movilización del colectivo, la revalorización de ellos mismos y del saber que poseían, y el afianzamiento de una dimensión política.

Ferrari y Cebey (2008) examinaron las formas de acción política que emergieron a partir de la organización en una cooperativa de una antigua fábrica, donde la posibilidad de agenciamiento permitió poner en cuestión la propiedad de la eficiencia (la cual era atribuida a los supervisores o jefes), rescatar y magnificar las trayectorias como grupo, e intercambiar saberes y tomar responsabilidades para afrontar colectivamente la organización y sus errores.

La referencia frente a lo que sucede con la subjetividad cuando los trabajadores toman a su cargo la empresa, ha sido explorada en profundidad por el trabajo adelantado por Fernández, López, Imaz y Calloway (2004). En este se señalan cambios en la producción de la subjetividad como son la redefinición del trabajo y de la condición de obreros, la transformación de las clásicas distinciones del mundo privado y del público, el establecimiento de nuevas estrategias de contacto con el mundo; también logran identificar la importancia de la dimensión de género en los casos donde fueron mujeres las que ocuparon las fábricas, allí se pudo evidenciar como las trabajadoras utilizaron prácticas domésticas en la lucha por conservarlas. En otro caso, Fernández, Imaz y Calloway (2005a) señalan una serie de tensiones que emergen en las dinámicas relacionales de las empresas recuperadas, las cuales tienen un efecto paradójico en la construcción de los colectivos, ya que, por una parte, actuaron como elementos diferenciadores que ayudaron a fortalecer la identificación con el grupo, pero, de otro lado, generaron rupturas desintegradoras. Estas tensiones emergieron por la heterogeneidad y las diferencias de los colectivos en cuanto a niveles de formación, por el rol anterior en la empresa (administrativo u operario), por la antigüedad en ella, por la situación o ciclo vital que se atraviesa, la participación o no en los antiguos sindicatos y, en los casos analizados, por estos autores de manera importante, por las ideas políticas; todas estas condiciones terminan produciendo "posicionamientos subjetivos muy distintos” (p. 185).

Un aspecto igualmente relevante fue la transición hacia la creación de "fábricas sin patrón", que generó una ruptura con la sumisión disciplinaria del obrero establecida desde la división patrón y asalariado e institucionalizada en las empresas que 
funcionaban bajo la lógica del capital. Frente a esta condición, emergen diversos posicionamientos personales y colectivos que transforman los vínculos en relaciones más cercanas con sus antiguos compañeros, debido a la ruptura del control y de la disciplina tayloristas propias de la fábrica. Este cambio permitió la identificación ya no como una familia, sino, en algunos casos, como una comunidad (Fernández, Imaz y Calloway, 2005b). Los cambios se evidencian "en el posicionamiento subjetivo respecto a sí mismos, sus pares, a la organización y a las funciones que desempeñan allí y que les exige un manejo de códigos y prácticas que por su experiencia anterior les resultaban ajenos" (Fernández et al., 2004, p. 181).

En el caso colombiano, la entrega de las empresas a los trabajadores respondió a otros intereses y mecanismos que limitaron el agenciamiento de los trabajadores en este proceso. La crisis económica de la década de 1990, los cambios de los modelos de producción y los malos manejos administrativos, llevaron al país a una situación en la cual muchas empresas se vieron abocadas al cierre, una alta proporción del sector manufacturero de las principales ciudades del país, donde estaba concentrado el proceso de industrialización (Danies, 2004).

Desde la Constitución Política de $1991^{1}$, ya se avistaban posibilidades de permitir una participación más protagónica de los trabajadores en los procesos de recuperación empresarial, pero son pocos los casos documentados. Respondiendo a la situación de crisis, el Gobierno nacional, expidió en el año 1999 la ley 550, la cual buscó favorecer que las empresas no se liquidaran definidamente y que pudieran seguir en funcionamiento, para atender las obligaciones que adeudaban, e intentar salvarse y evitar el cierre definitivo, el cual impactaría de manera significativa el problema del desempleo. La ley también incluyó la posibilidad de que los traba-

1 "Artículo 60. El Estado promoverá de acuerdo con la ley el acceso a la propiedad. Cuando el Estado enajene su participación en una empresa, tomará las medidas conducentes a democratizar la titularidad de sus acciones, y ofrecerá a sus trabajadores, a las organizaciones solidarias y de trabajadores, condiciones especiales para acceder a dicha propiedad accionaria". (Constitución Política de Colombia, 1991) jadores accedieran a la propiedad de la empresa, abriendo las puertas para que la recibieran como parte de pago de los pasivos que les adeudaba; esta estrategia se conoció como dación en pago.

Cuando la empresa era declarada en quiebra, la Superintendencia de Sociedades nombraba un liquidador, figura encargada de hacer los pagos a los acreedores, someter a subasta pública los bienes de la empresa y cuando era posible, promover la constitución de nuevas sociedades por parte de los trabajadores que ahora eran accionistas de la empresa (acciones representadas en maquinaria, mobiliario, edificaciones, terrenos, etc.). La entidad oficial brindaba capacitación en aspectos como el manejo de la empresa, la resolución de los conflictos, además de realizar vigilancia a las actividades desarrolladas, ya que en este tránsito muchos procesos se informalizaron. Las presiones a los liquidadores por parte de los diferentes actores involucrados, no siempre crearon escenarios para que los trabajadores se quedaran con la empresa.

Para el caso colombiano, la entrega fue mediada por una serie de mecanismos de trámite legal que muchas veces hicieron que el proceso liquidatorio durara varios años, período en el cual se iba descapitalizando la empresa y en el que algunas fábricas llegaron a ser insostenibles. En muchos de los casos, la iniciativa de conformar la empresa respondió más al interés del empresario o del liquidador en continuar con el funcionamiento de estas, las cuales en ocasiones eran empresas líderes en su sector y en el país. Un apoyo importante para la consolidación de estas nuevas sociedades fueron los procesos formativos y de consultoría 2 .

De otro lado, en la indagación realizada en el sector rural, se encontraron mecanismos diferentes a la ley 550 para entregar la empresa a sus trabajadores; estas alternativas fueron implementadas por parte de los antiguos dueños quienes optaron por liquidar la empresa, cuando vieron disminuido su margen de ganancia porque la empresa había

2 Ofrecidos por el programa de "Apoyo para la reactivación de empresas cuyos trabajadores reciben activos en dación de pago" desarrollado por el Instituto Pensar de la Pontificia Universidad Javeriana desde el año 2003, los cuales fueron dirigidos a $20 \mathrm{em}$ presas de diversas regiones del país. 
dejado de ser lo suficientemente rentable, razón por la cual optaron por ceder el negocio a los trabajadores, sin declararse en quiebra. La negociación directa entre el empresario y los trabajadores y la constitución de una cooperativa de trabajadores, fue un camino para la entrega de la empresa. Finalmente, en las investigaciones realizadas sobre las empresas recuperadas en Colombia, se encontró un caso en el cual los trabajadores decidieron "tomarse pacíficamente" las instalaciones de la empresa y desde allí presionar al Gobierno para ponerla nuevamente a funcionar, a pesar de la orden de cierre que se había declarada. Luego de la toma, se utilizó la vía de la contratación directa con el Estado (a través de la ley $80^{3}$ ).

\section{Metodología}

La propuesta metodológica planteó un proceso interdisciplinar cualitativo desde un diseño de estudio de caso, que permitiera estudiar las formas particulares de un caso singular o único (los trabajadores), enfrentado a una situación en particular (recuperación de la empresa) (Stake, 2007). Como técnicas de recolección de la información se realizaron 5 grupos focales con los trabajadoresaccionistas y un total de 14 entrevistas individuales semiestructuradas con trabajadores-accionistas, gerentes y expertos. Además, se analizaron documentos como actas de asambleas en todas las empresas, informes de investigación sobre las empresas participantes realizados por otros actores ${ }^{4}$ (5) y publicaciones locales sobre los casos de las empresas (2). El análisis de la información se realizó a través de la interpretación directa (Stake, 2007) de los textos recogidos durante el trabajo de campo.

Debido al diseño elegido, no hubo muestreo, sino una preselección de los casos de estudio teniendo en cuenta su disponibilidad y el acceso a

3 Cada trabajador puede constituir una empresa unipersonal, y luego si se requiere puede asociarse con otros en uniones temporales, para ofrecer un servicio que pueda ser contratado por una entidad del Estado.

4 Cuatro informes del Proyecto Flexibilización y Bienestar Laboral de la Facultad de Psicología de la Pontificia Universidad Javeriana (2005-2008) y uno de la Universidad de San Gil (Santander, Colombia). la información solicitada desde el consentimiento informado, como lo sugiere Stake (2007). Las empresas participantes fueron las siguientes.

\section{Empresa 1}

Ubicada en zona urbana. En el 2005, a través de la ley 550, se constituyó como Sociedad Anónima, con un socio mayoritario con cerca del $48 \%$ de las acciones. Manufactura y comercializa calzado. Tiene 11 socios vinculados como trabajadores de la planta, cada uno participa con el 1,5\% de la sociedad, y tiene antigüedad entre 16 y 24 años. La participación femenina constituye el 88 \% del total de trabajadores, y la edad promedio está en 57,8 años.

\section{Empresa 2}

Ubicada en zona urbana. Se acogió a la ley 550 y se constituyó como Sociedad Anónima en el año 2003; un socio cuenta con $70 \%$ de la participación accionaria. Fabrica y comercializa insumos eléctricos y electrónicos; 13 socios son trabajadores, la edad promedio es de 46 años y el $69 \%$ son hombres, mientras que la antigüedad oscila entre 16 y 27 años.

\section{Empresa 3}

Ubicada en zona rural. Fue entregada por los dueños luego de una liquidación directa y se constituyó en cooperativa multiactiva; por esta razón, los trabajadores cuentan con la misma participación accionaria. Manufactura fibras naturales de industrialmente; tiene 103 asociados como trabajadores de los cuales el $74 \%$ son hombres; la edad promedio es de 47 años.

\section{Empresa 4}

Ubicada en zona rural. Fue tomada por parte de los trabajadores como presión al Gobierno para su entrega a los trabajadores y se convirtió en Cooperativa de Trabajo Asociado en el 2003. Ofrece servicios de administración y logística hotelera. 
Cuenta con 12 asociados cuya edad promedio es de 49 años, siendo las mujeres el 75\%. El 75\% tiene una antigüedad de más de 17 años.

\section{Resultados}

El análisis mostró aspectos diferenciales frente al contexto, señalando la importancia en las dimensiones de lugar, espacio y tiempo para configurar las transformaciones en la subjetividad de los trabajadores. Estas condiciones son: a) empresas ubicadas en zonas urbanas industriales de Bogotá, que se acogieron a ley 550 para enfrentar la crisis y que adquirieron la forma de Sociedad de Capital Anónima en la nueva empresa y b) empresas ubicadas en contextos rurales, que no se acogieron ley 550 para enfrentar la crisis y que asumen la forma cooperativa en la nueva sociedad. Como lugares de configuración de la subjetividad, se pueden enunciar de manera transversal en las empresas participantes las siguientes categorías: la reflexión sobre la experiencia misma, la generación de un nuevo colectivo, las nuevas formas de relación y la experiencia de convertirse en dueño.

\section{Empresas ubicadas en zonas urbanas industriales de Bogotá}

Se acogieron a ley 550 para enfrentar la crisis y adquirieron la forma de sociedad de capital limitada o anónima en la nueva empresa.

\section{La reflexión sobre la experiencia misma}

La nueva condición condujo a los trabajadores a reflexionar sobre lo que había sido su tránsito en las antiguas empresas. Esto derivó, para algunos, en una valoración positiva de dicha experiencia, ya que el haber estado allí por varios años les permitió desarrollar proyectos vitales que en su condición de asalariados habría sido difícil de alcanzar por fuera de una vinculación laboral (compra de una vivienda para darle seguridad a sus familias o acceso a educación tanto para ellos como para sus hijos). No obstante, otros valoraron su paso por la antigua empresa en términos más negativos: la crisis que generó la quiebra les permitió una toma de conciencia del tiempo perdido en esos años de trabajo al estar dependientes de un salario y de conformarse solo con lo que este trabajo les ofrecía, reflexión que les condujo a repensar la propia vida en su condición de dependencia como obreros y de las pocas posibilidades de ejercer la agencia que tienen; el cambio a la nueva empresa no transformó su situación de dependencia.

Estas connotaciones otorgadas al enfrentarse a la experiencia de recuperar la antigua empresa, posicionaron de manera diferencial las subjetividades que allí se construyeron y aunque se reconoce la libertad que tuvieron para elegir si se iban o se quedaban, también son conscientes de que esa libertad estuvo restringida a su condición de ser un asalariado en riesgo de perder su única fuente de ingreso. El paso de ser trabajador a ser accionista, en muchos casos se asumió por parte de los trabajadores como una decisión no voluntaria, en la cual no tuvieron posibilidades de ejercer su agencia. Paradójicamente, a pesar de ser obreras y obreros con poca formación, esta situación no había sido considerada como un factor de desventaja en la antigua empresa, pero al enfrentarse a la posibilidad de ser excluidos del mundo laboral emergió una toma de conciencia de su vulnerabilidad para ingresar a otros circuitos de producción. En el caso de la Empresa 1, las trabajadoras comenzaron a describirse como mujeres cabeza de familia, sin mayores oportunidades en el mercado laboral, por su edad y nivel de formación, comprensión que desde la carencia las impulsó a involucrarse en el proyecto de constitución de la nueva sociedad, por no tener otras opciones. En este escenario de no acción, algunos trabajadores se percibieron sometidos ya que no contaron con la suficiente información y compresión de los aspectos legales y financieros del proceso de liquidación, y de otro lado engañados, frente al pago del valor real de las acreencias con la maquinaria ofrecida.

\section{La generación del colectivo}

La experiencia de crisis les permitió la construcción de un nosotros compartido, enunciado desde su nueva condición de trabajadores y socios. 
Los trabajadores han transformado la manera de enunciarse o nombrarse a ellos mismos, aparecen denominaciones como "socio-trabajador", o "trabajador-accionistas" que, en general, muestran un empoderamiento o toma de conciencia: el rótulo de socio les ha permitido a los antiguos trabajadores, más que entenderse a sí mismos como dueños, entenderse como un grupo de personas que comparte una experiencia en común. Esta identificación fue posible en un nosotros convocado por estar en una historia compartida (la vida en la empresa y la quiebra), como poseedores de un saber empírico valioso, de reconocerse en esta experiencia como dependientes de un salario (sin otras formas de producir capital y asumiendo algunas condiciones laborales precarias), por las redes de relación anteriores existentes como trabajadores (compartir la condición de asociados de una fondo de empleados o haber pertenecido al sindicato), lo cual generó sentimientos de confianza, apoyo y compasión de la propia situación.

La nueva empresa les ha permitido fortalecer las relaciones de amistad que venían cultivando desde hace años y la apropiación de ella, donde la metáfora de la familia actúa como elemento de cohesión y como trasfondo explicativo de algunos conflictos que allí suceden. El haber compartido una historia común por 10 o 20 años de trabajo y la percepción de haber trabajado en la mejor empresa del sector (que no debe desaparecer), fortalecieron los esfuerzos por conformar el colectivo y les generó una sensación de continuidad entre las historias de las empresas (la antigua y la nueva). En el caso de la Empresa 1, la visión de las trabajadoras como colectivo de iguales se expresa en actividades de cuidado mutuo y cooperación entre las diferentes socias trabajadores, la toma de meriendas compartidas, la preparación del café de la mañana, la generación de ayudas a quien tenga alguna dificultad familiar; la presencia de lo femenino se expresa en el importante lugar del cuidado maternal en las relaciones laborales.

\section{Las formas de relación}

La generación de este colectivo ha permitido cambiar la obediencia por la confianza en sus relaciones; las estrategias de gestión implementadas han facilitado cierto grado de horizontalidad y de recuperación de la confianza que se había perdido en el proceso liquidatorio. Esta situación también les ha permitido preguntarse de qué manera como socios pueden reclamar más igualdad tanto en la participación como en la toma de decisiones. La tenencia de unas acciones de capital las empoderó para reclamar salarios más equitativos y justos con el trabajo realizado, además para demandar reconocimiento a la hora de decidir hacia donde se dirige la empresa. Aparecen nuevas divisiones del colectivo, los "de afuera y los de adentro", que muestran los límites del territorio de relación marcados por el lugar de actuación de los trabajadores que siguieron vinculados en esta condición y los que solo son socios, señalando un reclamo a estos últimos: "los que luchamos de frente a los problemas y los espectadores" y reconociendo una transformación de su condición de trabajador, ahora más comprometido y que siente esta nueva empresa como suya.

\section{La experiencia de ser dueño}

El cambio de trabajador a socio, aunque les ha generado otras posibilidades de relación entre ellos mismos y con la Gerencia, no ha sido suficiente para lograr una mayor apropiación de lo que significa ser dueño. La experiencia de sentirse propietario, resultó secuestrada por la distribución de la participación accionaria que ellos tienen y la que tienen los socios mayoritarios, las limitadas posibilidades de decisión y la escasa generación de utilidades que ha dejado la empresa que no permiten lograr un distanciamiento de lo que eran antes, cuando no eran socios, lo cual genera sentimientos paradójicos: "cuando no éramos accionistas ganábamos mucho más, ahora que somos accionistas, ganamos menos... de socio solo el nombre, pues la plata no se ve". 
Esta situación los ha puesto en constantes tensiones sobre la participación y la decisión, pues a pesar de querer involucrarse de manera más activa en los procesos que se han adelantado en la constitución de la nueva empresa, la inequidad en la decisión no ha generado posibilidades reales de ejercer un rol más activo como socio. Como trabajador la situación ha sido diferente, ya que sienten que deben pelear y luchar por conservar sus puestos de trabajo. Los procesos formativos realizados, el sostenimiento de la nueva empresa y la retroalimentación recibida por sus grupos de referencia, los ha llevado a definir los espacios de la agencia que tienen ellos mismos en su condición de socios trabajadores, esto es, lo que pueden gestionar ellos mismos en sus puestos y áreas de trabajo, para sacar adelante la empresa.

Un elemento diferencial en el grupo de trabajadores de la Empresa 2, lo constituye el paso por la experiencia sindical; quienes estuvieron más involucrados ya sea porque fueron directivos o tuvieron un papel más activo en este, refieren de manera más evidente el deseo de ser empresarios. Para ellos, la liquidación de la empresa les abrió oportunidades de acceder a la propiedad de la fábrica, de ser dueños, situación que de otra manera habría sido imposible para ellos como obreros.

\section{Empresas ubicadas en contextos rurales}

No se acogieron ley 550 para enfrentar la crisis y asumen forma cooperativa en la nueva sociedad.

\section{La reflexión sobre la experiencia misma}

En las Empresas 3 y 4, la narración de su historia comienza con un hecho simbólico y significativo: la liquidación real de la empresa. Este hecho se hizo tangible en un cierre formal de la vinculación con la empresa anterior (negociación) y el pago de las prestaciones adeudadas con dinero en efectivo. Este proceso les permitió asumir la ruptura entre quiénes eran antes y de quiénes son ahora, luego de la conformación de la cooperativa. Al haber recibido el pago de la liquidación podían ahora decidir, con diferentes grados de libertad, si se quedaban o no. Las restricciones a la elección se posicionaron en problemas de empleo en la región y la existencia de otros espacios laborales que les permitieran poner en juego sus saberes.

En ambos casos, se muestra un mayor reconocimiento de las capacidades para transformar su entorno a partir de la acción colectiva. Logran tomar conciencia de que la lucha individual no les permitía avanzar y le apostaron a la creación de un sujeto colectivo, con mayor poder: en la Empresa 3, a partir de la conformación de una cooperativa de propiedad de los trabajadores, y en la Empresa 4 , con la toma de las instalaciones para presionar un cambio de decisión, reconociéndose capaces de recuperar las empresas y hacerlas viables a sus intereses. Esta ganancia de poder les generó mayor confianza como actores de cambio frente a la crisis.

En relación con la situación vital, la Empresa 3 reconoció que, en un alto porcentaje, sus asociados eran trabajadores jóvenes con mucha experiencia que podían ser útiles para la nueva Sociedad al construir sus proyectos vitales con la empresa a mediano y largo plazo, situación que les generó mayor empoderamiento; además se muestra de manera permanente el amor por su oficio y el interés por continuar allí. En la Empresa 4, otro lugar de enunciación se posicionó desde el ser mujer, con edades avanzadas, con poca calificación, sostenida en los años de trabajo para los empresarios, sin mayores oportunidades laborales y sin la posibilidad de contar con un nuevo empleo fuera de la empresa. No obstante, aparecen diversas posiciones de sujeto entre las mismas mujeres: unas buscando nuevas oportunidades para la empresa, otras esperando terminar su vida laboral alli "haciendo lo que toca", y otras que deciden estar mientras encuentran un nuevo trabajo más gratificante.

\section{La generación del colectivo}

En la construcción del colectivo, el fuerte arraigo expresado por los asociados de ambas cooperativas por conservar los patrimonios regionales (en la Empresa 3, la empresa insignia del municipio, en la Empresa 4 conservar un bien histórico) les impuso una obligación, a la hora de luchar por recuperar la 
empresa. Este colectivo, entonces, se pensó no solo desde la necesidad de conservar el ingreso, sino también de continuar sosteniendo parte de la historia común de la región y de permitir la jubilación de algunos de los trabajadores, cuidando de la crisis a los otros. La historia de ambas Cooperativas, ha sido reconocida por diferentes actores en su región y fuera de ella, como casos de éxito y sus asociados han sido connotados como ejemplos de lucha y actores centrales del desarrollo del municipio, lo que les ha permitido un mayor posicionamiento y una capacidad de transformación más alta como colectivo.

\section{Las formas de relación}

En la Empresa 3, se lograron subvertir las relaciones de subordinación entre administrativos y operarios, debido a la transformación radical de la estructura organizativa de la empresa (eliminación de cargos burocráticos y aplanamiento de la estructura), la disminución de las brechas salariales entre estos grupos, la rotación de puestos y la participación permanente de los operarios asociados en instancias de decisión como son el Consejo de Administración y la Asamblea, que han permitido configurar la idea de que "aquí todos los trabajos son importantes y valen lo mismo". No obstante, estas nuevas condiciones han generado rupturas en el colectivo entre operarios y administrativos, donde algunos llegan a manifestar intereses diferentes para la Sociedad, lo que ha generado un clima de permanente tensión y conflicto. En estas condiciones, se ha vuelto poco deseable la promoción hacia cargos administrativos.

En la Empresa 4, se han conservado las jerarquías en la relación del poder en la que se configura un trabajador subordinado, lógicas que son retroalimentadas por el contexto de la región, donde la forma hacienda es aún hegemónica y porque se ha centralizado la toma de decisiones en la Gerencia. En su rol de asociados, los trabajadores no se han enfrentado a experiencias diferenciales en relación con lo que hacían antes de serlo; esto dificulta su empoderamiento frente a su nuevo rol y a la toma de decisión. Se evidencia una división del colec- tivo: "los de adentro" y "los de afuera", pero aquí se relaciona con las diferentes posturas frente al proyecto de la cooperativa. Los primeros son activos, pero en su rol de trabajadores más que como asociados son, en su mayoría, personas mayores que atienden las labores de la cocina y servicios de mantenimiento de la Cooperativa, mientras que los segundos, son quienes han logrado desarrollar más su papel de asociados, aportando ideas, vinculándose a los nuevos negocios de manera activa, asumiendo cargos administrativos y son quienes ven más a largo plazo su futuro allí.

\section{La experiencia de ser dueño}

En la Empresa 3, la experiencia les ha permitido a muchos sentirse dueños. Por una parte, han logrado un crecimiento económico suficientemente alto como para generar utilidades, que al ser redistribuidas les han permitido iniciar a los asociados proyectos de inversión individuales o familiares para disponer de otro ingreso y disminuir la relación de dependencia con el puesto de trabajo, como única fuente de ingresos. De otro lado, los mecanismos participativos contemplados por el modelo cooperativo han permitido que tomen decisiones y que puedan legitimar y validar su conocimiento experto como obreros, para sacar adelante la empresa. De acuerdo a los estatutos de la Cooperativa, su participación puede cederse a algún miembro de la familia, lo que les permite, simbólica y realmente, heredar su patrimonio, a pesar de que las máquinas de la planta sean de la Cooperativa.

En la Empresa 4, la experiencia de ser dueño ha sido asumida por algunos que están permanentemente en la búsqueda de nuevas oportunidades, pero otros siguen considerándose a sí mismos solo con capacidad de aportar a la empresa desde su trabajo cotidiano, para sostener el ingreso y poder así atender a las necesidades de sus familias. No obstante, lo que se valida aquí es la condición de asociado, ya que les permite conservar el puesto de trabajo, sin estar expuesto a la presión de quedarse desempleado y les posibilita a algunos llegar a obtener su jubilación. Otro aspecto que han podido transformar es el acceso a la educación; en este 
caso, se promueve la alfabetización y los estudios de bachillerato para todos sus trabajadores, lo que mejora el acceso y participación en los espacios en los cuales se relacionan.

\section{Discusión}

Asumir la recuperación de la empresa en la que trabajaban implicó para los trabajadores involucrados en los casos revisados, una serie de transformaciones tanto en la comprensión de sí mismos como en la manera en que se relacionan con su entorno social y económico. En esta investigación, se pudo evidenciar que la forma asumida por la nueva sociedad - cooperativa o sociedad de capital-se relaciona de manera importante con las posibilidades que encuentran los trabajadores para ejercer su agencia, poder comprenderse a ellos mismos de manera diferencial y transformar su entorno. En los casos revisados, se puede observar una constante lucha por resistirse a los nuevos ejercicios de dominación que pueden ejercer los socios mayoritarios, por continuar en la búsqueda de romper con la dependencia al puesto de trabajo y con el estereotipo del obrero que se construyó en los desarrollos del proyecto modernizador en el país.

De otra parte, el espacio donde está ubicada la empresa (urbana o rural) tiene un lugar relevante en la construcción del nosotros; en el caso de lo rural, la región tiene un rol central y se vincula con las posibilidades de sostener un colectivo donde emergen otros aspectos para la unión, diferentes a la conservación del puesto de trabajo. En los casos urbanos, el nosotros está referido a la fábrica, la fragmentación y anonimato que genera la ciudad se muestra en los procesos de individualización a los que han sido expuestos, donde se sienten más solos en la lucha. Un elemento importante en las transformaciones de la subjetividades en los casos revisados, fue el tiempo que tomó el proceso de liquidación, de donde podemos concluir que en las empresas donde se utilizó la ley existente para la entrega a los trabajadores, el proceso se hizo más largo, lo cual permitió que se debilitara la confianza y se perdiera capacidad para la transformación. En este sentido, para favorecer que la experiencia se convierta en oportunidad, quizás resulte más adecuado que la entrega de la empresa se haga de manera directa, donde medien los intereses de menos actores y donde los trabajadores puedan sentirse con mayor poder en las negociaciones.

En acuerdo con Castañeda (1997) y Fernández, Imaz y Calloway (2004), fue posible identificar diversas posiciones de sujeto. Las subjetividades de estos trabajadores se ven tejidas por lo que ha sido su historia en la sociedad (dibujada muchas veces desde la precariedad) y en sus antiguas empresas, el género y la edad. Frente a los casos de empresas recuperadas revisados en la literatura, se logran determinar elementos de convergencia y diferenciales con el movimiento social del Cono Sur latinoamericano. La experiencia subjetiva de lucha, el esfuerzo por continuar y no excluirse del sistema productivo y las nuevas rupturas del colectivo, aparecen como aspectos convergentes, que permiten establecer algunas conclusiones sobre cómo la experiencia de recuperar una empresa genera la transformación de las subjetividades de los trabajadores involucrados en ella. Sin embargo, un aspecto diferencial, tiene que ver con la ausencia de un movimiento colectivo social que evidencie nuevas prácticas relacionales, donde se revalorice el ser trabajador y que facilite una transformación de las relaciones de subordinación y de poder, institucionalizadas en la configuración del obrero. La necesidad de construir relaciones donde haya más participación y democracia en el acceso a la propiedad, de generar desde su nueva condición de accionistas condiciones que promuevan la independencia del puesto de trabajo y revaloricen su trabajo (tanto social como económicamente), son algunos de los aspectos que hacen de la experiencia de recuperar una empresa, una oportunidad de transformación.

\section{Referencias}

Arango, L. (1992). Mujeres obreras, familia y políticas empresariales: la historia de Fabricato. Bogotá: Universidad de los Andes.

Archila, M. (1991). Cultura e Identidad Obrera. Bogotá: Cinep. 
Bialakowsky, A., Grima, J., Costa M. \& López, N. (2005). Gerencia de empresas recuperadas por los trabajadores en Argentina. Revista Venezolana de Gerencia, 10 (31), 359-384.

Bialakowsky, A., Robledo, G., Grima, J., Rosendo, E. \& Costa M. (2004). Empresas recuperadas: cooperación y conflicto en las nuevas formas de autogestión de los trabajadores. Revista Venezolana de Gerencia, 9 (26), 229-253.

Castañeda, W. (1997). Ser obrero en la industria. En A. Weiss (Ed.), Modernización industrial: empresas y trabajadores (pp. 379-442). Bogotá: Universidad Nacional de Colombia.

Cabruja, T. (Ed.), Albertín, P., Garay, A. \& Gordo, A. (2005). Psicología: perspectivas deconstruccionistas, subjetividad, psicopatología y ciberpsicología. Barcelona: UOC.

Constitución Política de Colombia (1991). Descargado el 10 de octubre de 2008, de http://www.personeriabogota.gov.co/index.php?idcategoria $=1107$.

Danies, R. (2004). Causas de la liquidación obligatoria de sociedades: estrategias para prevenir las crisis. Bogotá: Superintendencia de Sociedades.

Dávila, C. (2001). Teorías organizacionales y administración. Enfoque crítico (2⿳亠丷⿵冂⿱丷口一亅 ed.). Bogotá: McGraw Hill.

Dávila, R, Huertas, O y Castillo, D. (2010) Estrategias de gestión seguidas por los trabajadores de empresas recuperadas; el caso de dos cooperativas rurales en Colombia. En: A. Urbiola (Ed.), Ventaja cooperativa y organización solidaria en un mundo complejo. Estrategias cooperativas frente a la ambigüedad e incertidumbre (pp. 213-242). México: Fontamara.

Deledicque, L., Féliz, M. \& Moser J. (2005). Recuperación de empresas por sus trabajadores y autogestión obrera. Un estudio de caso en una empresa en Argentina. CIRIEC-España, Revista de Economía Pública, Social y Cooperativa abril 051, 51-76.

Fernández, A. (2005). Notas para la constitución de un campo de problemas de la subjetividad. En A. M. Fernández (Ed.), Instituciones Estalladas (pp. 37-57). Buenos Aires: Eudeba.

Fernández, A., Imaz, X. \& Calloway, C. (2005a). La capacidad productiva de las tensiones. En A. Fernández (Ed.), Política y Subjetividad. Asambleas barriales y fábricas recuperadas (pp. 185-200). Buenos Aires: Biblos.
Fernández, A., Imaz, X. \& Calloway, C. (2005b) La invención de las fábricas sin patrón. En A. Fernández (Ed.), Política y Subjetividad. Asambleas barriales y fábricas recuperadas (pp. 201-242). Buenos Aires: Biblos.

Fernández, A., López, M., Imaz, X. \& Calloway, C. (2004). Brukman: de las estrategias de supervivencias a la gestión. En A. Fernández (Ed.), Política y Subjetividad. Asambleas barriales y fábricas recuperadas (pp. 161-184). Buenos Aires: Biblos.

Ferrari, C. (2008). Tiempos de incertidumbre: causas y consecuencias de la crisis mundial. Revista de Economía Institucional, 10 (19), 55-78.

Ferrari, L. \& Cebey, M. (2008). Nuevas formas de acción política: el caso de las empresas recuperadas por sus propios trabajadores. Athenea Digital, 10, 90-102.

Fontela, E. (2007). Cooperativas que recuperan empresas y fábricas en crisis. Buenos Aires: Manuscrito no publicado.

González, F. (2002). Sujeto y subjetividad. México: Internacional Thomson Editores.

Graham, C. \& Pettinato, S. (2004). Felicidad y Penurias. Oportunidades e inseguridad en las nuevas economías de mercado. Buenos Aires: Prometeo.

Guerra, P., Martí, J. \& Amorín, C. (Eds.). (2004). Empresas recuperadas, entre la reflexión y la práctica. Montevideo: Nordan-Comunidad.

Halford, S. \& Leonard, P. (2006). Place, space and time: Contextualizing workplace subjectivities. Organization Studies, 27, 657-676.

Huertas, O. (2007). Las incertidumbres en el mundo del trabajo contemporáneo. Cuadernos de Psicología, 3 (2), 33-48.

Martí, J. (2007). Globalización, transformaciones en el mundo del trabajo y cooperativa de trabajadores. La recuperación de empresas en Uruguay y Argentina. En M. Radrigán (Coord.), El Rol de las cooperativas en un mundo globalizado (pp. 182-199). Sherbrooke, Canadá: Irecus.

Pulido, H. (2007). Produciendo trabajadores modernos: conocimiento psicológico y el mundo del trabajo en el sur. Universitas Psychologica, 6 (1), 27-38.

Stake, R. (2007). Investigación con estudios de caso (4⿳⺈⿴囗十一 ed.). Madrid: Morato. 
Serrano, A., Moreno, F. \& Crespo, E. (2001). La experiencia subjetiva del trabajo en una sociedad en transformación. En E. Agulló \& A. Ovejero (Coords.). Trabajo, individuo y sociedad. Perspectivas psicosociológicas sobre el futuro del trabajo (pp. 4964). Madrid: Pirámide.
Sennett, R. (2006). La cultura del nuevo capitalismo. Barcelona: Anagrama.

Weiss, A. (1997) Modernización industrial: empresas y trabajadores. Bogotá: Universidad Nacional de Colombia. 